\title{
SULLO SVILUPPO \\ D’UNA FUNZIONE UNIFORME DI VARIABILE COMPLESSA, DOTATA DI SINGOLARITA ISOLATE, IN SERIE COLLE CARATTERISTICHE SEPARATE.
}

\author{
Nota di F. Bucca, in Palermo.
}

Adunazza del 28 febbrajo 1897 .

I. Dato nel piano della variabile complessa un insieme d'infiniti punti isolati, colle affisse $a_{0}, a_{x}, \ldots a_{1} \ldots$, in guisa che si possono ordinare le quantità $a_{0}, a_{1}, a_{2} \ldots$ per ordine di grandezza dei loro moduli (disponendo in quell'ordine che si vorrà quelle di esse che han lo stesso modulo) $\left({ }^{*}\right)$, e data, corrispondentemente a ciascuno dei punti $a_{l}$, una serie di potenze intere e negative di $x-a_{l}$, senza termine costante, convergente in tutto il piano tranne che nel punto $a_{l}$; è noto $\left({ }^{*}\right)$ come si possa sempre costruire un'e-

(*) Volendo usare le denominazioni del Cantor a Beiträge zur Begrundung der transfiniten Mengenlehre „ (Math. Annalen, Bd. 46), diremo: E dato un insieme ordinato di punti nel piano, il cui tipo ordinatore è $\omega$, cioè un insieme la cui potenza è aleph-zero e tale che di due elementi $a_{\nu}, a_{\nu+1}$ ( $\nu$ è un numero cardinale finito) viene $a_{\nu}$ prima di $a_{\nu+1}$, quando $\left|a_{\nu}\right|<\left|a_{\nu+1}\right|$ e, nel caso di $\left|a_{y}\right|=\left|a_{y+1}\right|$, quando arg. $a_{\nu}<$ arg. $a_{y+1}$.

(*) Cfr. G. Mitt a g-I, e ff le r: "Sur la représentation analytique des fonctions monogènes uniformes d'une variable indépendante (Acta Mathematica, t. IV, 1884). 
SÚlio SVILUPPO D'UNA FÚNZIONE UNIFORME, ETTC.

spressicne analitica, la quale rappresenti una funzione analitica $f(x)$ avente per punti critici i punti $a_{0}, a_{1}, a_{2} \ldots$ colle caratteristiche $G_{0}\left(\frac{1}{x-a_{0}}\right), G_{1}\left(\frac{1}{x-a_{1}}\right), G_{2}\left(\frac{1}{x-a_{2}}\right) \ldots$, ció̀ una funzione che nell'intorno di ciascuno dei punti $a_{l}$ è rappresentabile sotto la forma

$$
f(x)=G_{l}\left(\frac{1}{x-a_{b}}\right)+p\left(x-a_{l}\right),
$$

essendo $\varphi\left(x-a_{l}\right)$ una funzione olomorfa nell'intorno di $a_{l}$. L'espressione è la seguente :

$$
f(x)=G_{0}\left(\frac{\mathrm{I}}{x}\right)+\sum_{l=1}^{\infty}\left[G_{l}\left(\frac{\mathrm{I}}{x-a_{l}}\right)-P_{l}(x)\right],
$$

in cui $P_{l}(x)$ son dei polinomi in $x$, i cui gradi, in generale, crescono con $l$, costruiti in guisa da rendere convergente, nell'intorno di ogni punto $x$ a distanza finita, la serie delle caratteristiche, ed in cui $G_{\mathrm{o}}\left(\frac{\mathrm{I}}{x}\right)$ indica la caratteristica nell'origine, che si suppone corrispondente al valore $a_{0}$.

E noto ancora che, in casi speciali, possono esistere diverse leggi di formazione dei polinomi $P_{l}(x)$, e puó anche avvenire che possano scegliersi polinomi $P_{l}$ coi gradi tutti inferiori ad un numero finito, $i$ quali per conseguenza posson considerarsi tutti dello stesso grado finito, che noi vogliamo indicare con $m-\mathrm{I}$.

Quando ciò avviene, avremo dunque una serie, il cui termine generale è

$$
G_{l}\left(\frac{1}{x-a_{l}}\right)-P_{l}(x)
$$

dove $P_{l}$ è un polinomio variabile con $l$, ma di grado costante $m-$ I, e noi diremo tal serie uno sviluppo d'ordine $m$.

Si hanno sviluppi d'ordine finito, quando le singolarità nei 
puntii $a_{l}$ sone singolarita polari di ordine $p$ le pid̀ generali, pẹr guisa che si ha

$$
G_{l}\left(\frac{1}{x-a_{l}}\right)=\sum_{i=1}^{p} \frac{A_{l}^{(i)}}{\left(x-a_{l}\right)^{i}}
$$

ed esiste un numero finito $m$ tale che le serie

$$
\sum_{i=1}^{\infty}\left|\frac{A_{l}^{(i)}}{a_{l}^{m+1}}\right| \quad \quad(i=1,2, \ldots p)
$$

sian convergenti (*). Ma se le singolarità non sono singolarità polari, ovvero se, essendo singolaritì polari, l'ordine del polo dipende da $l$ in guisa da crescere indefinitamente al çrescere di $l$, ovvero se l'insieme è tale che non sia possibile trovare un numero finito $m$, pel quale le scrie $\sum_{l=1}^{\infty}\left|\frac{A_{l}^{(i)}}{a_{l}^{m+1}}\right|$ sian convergenti (come p. es. avviene șe $a_{l}=\log l$, ovvera $a_{l}=\log \log l, \ldots$ ed $A_{l}=\mathrm{r}$ ), allora non şi puo dire, senza ulteriore esame, se colle caratteristiche e coll'insieme dato possano formarsi sviluppi d'ordine funito.

2. I teoremi di Mittag-L effler e di Hermite sono molto utili quanda data una funzione uniforme $f(x)$ avente singolarita isolate, si voglia svilupparla in serie colle caratteristiche separate; con essi peró rimane incognita una parte dello sviluppo. Formando, infatti $_{3}$ le çaratteristiche per mezzo della nota formola di Laurent

$$
G_{2}\left(\frac{1}{x-a_{l}}\right)=-\frac{1}{2 \pi i} \int_{r_{l}} \frac{f(z)}{z-x} d z=\frac{1}{2 \pi i} \sum_{y=1}^{\infty}\left(x-a_{i}\right)^{-y} \int_{r_{l}}\left(z-a_{l}\right)^{\nu-1} f(z) d z,
$$

dove $\gamma_{l}$ è un cerchio descritto col centro in $a_{l}$ e di raggio sufficientemente piccolo perchè esso stia nell'intorno di $a_{l}$, si puó col procedimento del Mittag-Leffler, in ogni caso, e con quello di

(*) Hermite: “Sur quelques points de la théorie des fonctions 》. (Acta Soçietatis Sçientiarụm Fenniç, t. XII; Journal für Mathematik, Bd. 92). 
ŚULLO SVILUPPO D'UNA FUNZIONE UNIFORME, ETC.

Hermite, in casi speciali, formare un'espressione analitica $F(x)$, che ha le medesime singolarità della funzione $f(x)$; allora la differenza $f(x)-F(x)$, non avendo più singolarità a distanza finita, è una funzione intera che resta ignota e che bisogna determinare caso per caso.

Ci proponiamo di dare un metodo per la formazione, in generale, di tale funzione intera; $\mathrm{ma}$, appunta perche possono esistere della funzione data $f(x)$ parechi sviluppi, diversi, in serie colle caratteristiche separate, porremo la questione nei termini seguenti :

Data una funzione $f(x)$ uniforme, avente singolarità isolate, e data una legge di costruzione dei polinomi $P_{l}$ tali che la serie

$$
\sum_{l=1}^{\infty}\left[G_{l}\left(\frac{1}{x-a_{l}}\right)-P_{l}(x)\right]
$$

sia convergente uniformemente nell'intorno di ogni punto a distanza finita, diverso dai punti $a_{l}$, detirninare la funzione intera $g(x)$ che bisogna aggiungere ad essa affinchè sia

(1) $f(x)=g(x)+G_{0}\left(\frac{1}{x}\right)+\sum_{i=1}^{\infty}\left[G_{l}\left(\frac{\mathrm{I}}{x-a_{l}}\right)-P_{l}(x)\right]$.

S'intende che noi vogliamo riferirci a quei casi, in cui il metodo stesso per costruire $\dot{i}$ polinomi $P_{l}$ non costruisce $i_{a}$ funzione $g(x)$. Dimostreremo, infatti, in seguito $\left(n^{0} 6\right)$ che della funzione esiste uno sviluppo d'ordine non superiore ad $r+I$, quando si puó trovaŗe un numero finito $r$ in guisa che $\left|\frac{f(x)}{x^{r}}\right|$ si mantiene inferiore ad un numero fisso mentre $x$ si muove su una circonferenza qualunque d'una successione di circoli, che hanno i centri nell'origine ed $i$ raggi indefinitamente crescenti, e che non passano per alcuno dei punti singolari. Il metodo, che daremo per la formazione dei polinomi $P_{l}$, dà lo sviluppo completo della funzione senza l'indeterminazione di qualche sua parte.

3. Per metterci nel casa più generale, supponiamo che in ( $I$ ) 
il grado del polinomio $P_{l}$ sia $m_{l}-\mathrm{I}$, variabile con $l$, sicchè si abbia

$$
P_{l}(x)=\sum_{i=0}^{m_{l}-1} A_{l, \nu} x^{\nu}
$$

Pensiamo ad una successione qualunque di numeri positivi crescenti indefinitamente $R_{x}, R_{2}, \ldots R_{k} \ldots$, tale pero che sulle varie circonferenze $C_{1}, C_{2}, \ldots C_{k} \ldots$, che hanno il centro nell'origine e per raggi $R_{1}, R_{2}, \ldots R_{k} \ldots$, non cada alcuno dei punti $a_{l}$; dentro uno qualunque dei cerchi $C_{k}$ trovasi adunque un numero limitato di punti singolari per la funzione $f(x)$.

Partiamo dalla relazione

$$
f(x)=\frac{1}{2 \pi i} \int_{C_{k}} \frac{f(z)}{z-x} d z+\sum_{C_{k}} G_{l}\left(\frac{\mathrm{I}}{x-a_{l}}\right)
$$

in cui il segno sommatorio va esteso a tutte le caratteristiche relative ai punti $a_{l}$, compresa l'origine, che cadono dentro $C_{k}$, e che supporremo in numero di $n_{k}, x$ \& un punto qualunque interno a $C_{k}$ diverso dai punti $a_{l}$. Tale relazione è valida solo dentro $C_{k}$ e si ottiene colla considerazione che, avendo la funzione $f(x)$ dentro $C_{k}$ un numero finito di punti singolari, ed essendo continua al contorno, è lecito applicare il teorema dei residui di $\mathrm{Cau}$ chy.

$\mathrm{E}$ evidente che, se nella relazione precedente facciamo crescere indefinitamente $k$, potremo anche rappresentare la $f(x)$ sotto forma d'un limite

$$
f(x)=\lim _{k=\infty}\left[\frac{\mathrm{I}}{2 \pi i} \int_{C_{k}} \frac{f(z)}{z-x} d z+\sum_{C_{k}} G_{l}\left(\frac{\mathrm{I}}{x-a_{l}}\right)\right]
$$

e, sotto questa forma, $x$ puó essere un punto qualunque del piano, diverso dai punti $a_{l}$.

Questo modo di rappresentare la $f(x)$ è valido qualunque sia la funzione $f(x)$, purchè dotata di singolarità isolate. La funzione intera $g(x)$, che entra nello sviluppo (I), resterd adunque determinata dai due differenti modi (I) e (2) di rappresentare la $f(x)$; scri- 
SULLO SVILUPPO D'UNA FUNZIONE UNIFORME, ETC.

vendo la ( $\mathrm{I}$ ) nella forma

$$
f(x)=g(x)+G_{0}\left(\frac{I}{x}\right)+\lim _{k=\infty} \sum_{l=i}^{n_{k}}\left[G_{l}\left(\frac{I}{x-a_{l}}\right)-P_{l}(x)\right]
$$

e sottraendone la (2), si ricava :

$$
g(x)=\lim _{k \rightarrow \infty}\left[\frac{1}{2 \pi i} \int_{C_{k}} \frac{f(z)}{z-x} d z+\sum_{k=1}^{n_{k}} P_{l}\right]
$$

ovvero :

$$
g(x)=\lim _{k \rightarrow \infty}\left[\sum_{\nu=0}^{\infty} x^{\nu} \sum_{k=0}^{n_{k}} \sigma_{\nu+1, l}+\sum_{k=1}^{n_{k}} \sum_{\nu=0}^{m_{l}-x} A_{l, \nu} x^{\nu}\right]
$$

avendo all'integrale sostituito il suo sviluppo in serie, valido dentro al cerchio $C_{k}$, ed avendo indicato con $\sigma_{y+z, l}$ il residuo della funzione $\frac{f(z)}{z^{\nu+z}}$ nel punto $a_{l}$.

4. Supponiamo che lo sviluppo (I) della funzione $f(x)$ sia dell'ordine $m$; allora la doppia somma

$$
\sum_{k=1}^{m_{k}} \sum_{y=0}^{m_{l}-1} A_{l, \nu} x^{\prime \prime}
$$

viene ad essere la somma d'un numero finito di polinomi tutti dello stesso grado $m-\mathrm{I}$, e peró si può mettere sotto la forma d'un polinomio unico, i cui coefficienti son somme che, al crescere di $k$, diventan serie; cioè si puó rappresentare con

$$
\sum_{y=0}^{m=x} x^{y} \sum_{l=1}^{m k} A_{l, y}
$$

In tal caso la (3) si puó scrivere:

$$
g(x)=\lim _{k=0} \sum_{v=0}^{\infty} x^{\nu} \sum_{k=0}^{n_{k}}\left(\sigma_{v+1, l}+A_{h v}\right)
$$


convenendo di assumere

$$
\begin{aligned}
& A_{\mathrm{o}, \nu}=\mathrm{o} \quad \text { per tutti i valori di } \nu \text {, }
\end{aligned}
$$

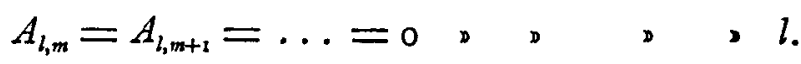

Ora io dico che si puo in (4) passare al limite termine a termine, e quindi si ha:

$$
\begin{gathered}
g(x)=\sum_{\nu=0}^{\infty} x^{\nu} \sum_{l=0}^{\infty}\left(\sigma_{y+1, l}+A_{h, \nu}\right) \\
=\sum_{y=0}^{m-1} x^{\nu} \sum_{l=0}^{\infty}\left(\sigma_{y+z, l}+A_{h, v}\right)+\sum_{\nu=m}^{\infty} x^{\nu} \sum_{k=0}^{\infty} \sigma_{y+1, l} .
\end{gathered}
$$

Infatti, derivando la (I) e la (I'), e designando con $f^{(\mu)}(x)$, $g^{(\mu)}(x), P_{l}^{(\mu)}(x), G_{l}^{(\mu)}(x)$ le derivate $\mu^{\text {ime }}$ rispetto ad $x$ di $f(x)$, $g(x), P_{l}(x), G_{l}\left(\frac{\mathrm{I}}{x-a_{l}}\right)$, si ha che la derivata $\mu^{\mathrm{ima}}$ di $f(x)$ e rappresentata in tutto il piano da

$$
f^{(\mu)}(x)=g^{(\mu)}(x)+G_{0}^{(\mu)}\left(\frac{\mathrm{I}}{x}\right)+\sum_{l=1}^{\infty}\left[G_{l}^{(i)}\left(\frac{\mathrm{I}}{x-a_{l}}\right)-P_{l}^{(\mu)}(x)\right]
$$

e dentro al cerchio $C_{\mathrm{k}}$ da

$$
f^{(\mu)}(x)=\frac{I}{2 \pi i} \int_{C_{k}} \frac{f(z)}{(z-x)^{\mu+1}} d z+\sum_{C_{k}} G_{l}^{(\mu)}\left(\frac{I}{x-a_{l}}\right) .
$$

Applicando poscia alla $f^{(\mu)}(x)$ lo stesso procedimento usato per la $f(x)$, si deduce :

$$
\begin{aligned}
g^{(\mu)}(x) & =\lim _{k=\infty}\left[\frac{1}{2 \pi i} \int_{C_{k}} \frac{f(z)}{(z-x)^{\mu+1}} d z+\sum_{l=1}^{n_{k}} P_{l}^{(\mu)}(x)\right] \\
& =\lim _{k=\infty} \frac{d^{\mu}}{d x^{\mu}}\left[\sum_{\nu=0}^{\infty} x^{\nu} \sum_{l=0}^{n_{k}}\left(\sigma_{\nu+s, l}+A_{l, \nu}\right)\right]
\end{aligned}
$$


SULLO SVILUPPO D'UNA FUNZIONE UNIFORME, ETC.

e di qui

$$
\frac{g^{(\mu)}(0)}{\mu !}=\sum_{i=0}^{\infty}\left(\sigma_{v+r, b}+A_{h, \nu}\right)
$$

per cui la $(\zeta)$ resta pienamente dimostrata.

Dalla (5) si ricava che la serie

$$
\sum_{i=0}^{\infty} \sigma_{v+2, l},
$$

cioè la somma dei residui della funzione $\frac{f(z)}{z^{\nu+1}}$ relativi al punto zero ed ai punti $a_{l}$ a distanza finita, è convergente quando $\vee \supseteq m$; e pero il minimo valore di $m$ non può essere inferiore al minimo valore di $v$, per cui la serie dei residui della funzione $\frac{f(z)}{z^{y+1}}$ è convergente; cioe non possono esistere polinomi $P_{l}$, di grado costante $m-\mathrm{I}$, tali che $m$ sia inferiore al detto valore minimo di $v$.

5. Cio posto, partendo dalla forma (2) di rappresentare la $f(x)$, che si pud scrivere :

$$
f(x)=\lim _{k=\infty}\left[\sum_{y=0}^{\infty} x^{\nu} \sum_{l=0}^{k_{k}} \sigma_{y+x, l}+\sum_{C_{k}} G_{l}\left(\frac{\mathrm{I}}{x-a_{l}}\right)\right]
$$

od ancora

$$
\text { (2") } f(x)=\lim _{k=\infty}\left\{\sum_{i=0}^{n_{k}}\left[G_{l}\left(\frac{I}{x-a_{l}}\right)+\sum_{\nu=0}^{m=z} \sigma_{y+1, l} x^{\nu}\right]+\sum_{k=m}^{\infty} x^{\nu} \sum_{l=0}^{k_{k}} \sigma_{v+z, l}\right\} \text {, }
$$

e notando che, se $f(x)$ ammette uno sviluppo d'ordine $m$; il limite

$$
\lim _{k \rightarrow \infty} \sum_{\nu=m}^{\infty} x^{y} \sum_{l=0}^{n_{k}} \sigma_{v+\mathrm{r}, l}
$$

esiste in virtú della $(5)$, ed è una funzione intera di $x$, si conclude per la $f(x)$ il seguente sviluppo:

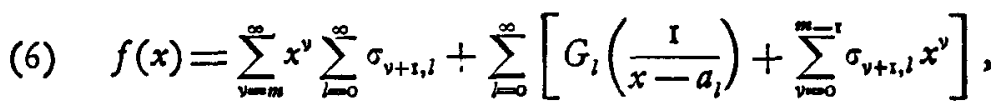

che è un particolare sviluppo d'ordine $m$.

Rend. Circ. Matem., t. XI, parte I $^{2}$.-Stampato il 19 marzo 1897. 
Dunque: Se una funzione uniforme $f(x)$ ammette uno sviluppo d'ordine $m$, caratterizzato dai polinomi $P_{l}$, essa ammetterà anche $l o$ sviluppo (6).

Tale sviluppo lo diremo principale, in quanto che, se la funzione non ha lo sviluppo principale (6), non puob ammettere certamente altri sviluppi d'ordine finito.

Percio le condizioni necessarie e sufficienti, alle quali deve soddisfare una funzione uniforme, dotatà di singolarità isolate, affinchè di essa esista uno sviluppo d'ordine finito, si riducono alle condizioni di convergenza dello sviluppo (6).

Quindix tenendo presente la formola $\left(2^{\prime \prime}\right)$ i si ricava che una funzione uniforme $f(x)$ dotata di singolarita isolate, ammette uno sviluppo in serie d'ordine finito $m$ allora e solo allora quando la serie

$$
\sum_{v=m}^{\infty} x^{y} \sum_{l=0}^{\infty} \sigma_{v+r, l}
$$

converge in tutto il piano uniformemente.

6. Passiamo a dimostrare il seguente teorema:

Se una funzione $f(x)$, uniforme, con singolarità isolate, è tale che esiste un numero finito ir per cui

$$
\left|\frac{f(x)}{x^{n+1}}\right|
$$

si mantiene inferiore ad un numero finito $M$, quando $x$ si muove sulle circonferenze $C_{k}$, la funzione $f(x)$ ammette lo sviluppo principale d'ordine $r+\mathrm{r}$.

Invero, l'integrale

$$
\frac{1}{2 \pi i} \int_{C_{k}} \frac{f(z)}{z-x} d z
$$

dentro al cerchio $C_{k}$ si sviluppa in serie di potenze di $x$, la quale, arrẹstata alla potenza $x^{r+1}$, dà : 
SULLO SVILUPPO D'UNA FUNZIONE UNIFORME, ETC.

$$
\begin{gathered}
\frac{I}{2 \pi i} \int_{C_{k}} \frac{f(z)}{z-x} d z=\frac{i}{2 \pi i} \int_{C_{k}} \frac{f(z)}{z} d z+\frac{x}{2 \pi i} \int_{C_{k}} \frac{f(z)}{z^{2}} d z+\ldots \\
\quad \ldots+\frac{x}{2 \pi i} \int_{C_{k}} \frac{f(z)}{z^{r+1}} d z+\frac{x^{r+1}}{2 \pi i} \int_{C_{k}} \frac{f(z)}{z^{r+2}}\left(1+\varepsilon_{r+1}\right) d z,
\end{gathered}
$$

ove $\varepsilon_{r+1}$ è una quantità dipendente da $x, z, r$, tale però chè, fissato $x$ comunque, e qualunque sia $r$, pei valori di $z$ di modulo superiore ad un certo $N>0$, convenientemente scelio, e $\left|\varepsilon_{r+1}\right|<n$ ( $n$ essendo un numero positivo dato a priori). Quindi, considerando le circonferenze $C_{k}$ di raggio maggiore di $N$, si ha

$\left|\frac{x^{r+1}}{2 \pi i} \int_{C_{k}} \frac{f(z)}{z^{r+2}}\left(\mathrm{~s}+\varepsilon_{r+1}\right) d z\right|<\frac{|x|^{r+1}}{2 \pi} \int_{C_{k}} \frac{M}{|z|^{2}}(\mathrm{r}+\eta) d s=\frac{|x|^{r+z}}{R_{k}} M(\mathrm{r}+n) ;$

ma per $k=\infty, \lim R_{k}=\infty$; dunque si ha

$$
\lim _{k=\infty} \frac{x^{r+i}}{2 \pi i} \int_{C_{k}} \frac{f(z)}{z^{r+i}}\left(1+\varepsilon_{r+1}\right) d z=0
$$

qualunque sia $x$; val quanto dire che la serie

$$
\sum_{v=r+2}^{\infty} x^{\nu} \sum_{i=0}^{n k} \sigma_{y+1, l}
$$

per $k=\infty$ ha per limite zero qualunque sia $x$; e perb si ha

$$
\sum_{i=0}^{\infty} \sigma_{v+r_{2} l}=0
$$

quando $v=r+\mathrm{I}, r+2, \ldots$

Dalla $\left(2^{\prime \prime}\right)$ ricaviamo dunque, ponendo $m=r+\mathrm{I}$,

$$
\begin{aligned}
& f(x)=\lim _{n \rightarrow \infty} \sum_{i=0}^{n_{k}}\left[G_{l}\left(\frac{1}{x-a_{l}}\right)+\sum_{y=0}^{r} \sigma_{y=1} x_{t=1}^{x^{v}}\right] \\
& =\sum_{y=0}^{r} \sigma_{y+r, 0} x^{\nu}+G_{0}\left(\frac{\mathrm{r}}{x}\right)+\sum_{i=1}^{\infty}\left[G_{l}\left(\frac{1}{x-a_{i}}\right)+\sum_{\nu=0}^{i} \sigma_{\nu+r_{l},} x^{\nu}\right] \text {, }
\end{aligned}
$$

che è lo sviluppo principale d'ordine + Ir 
7. Applicheremo le considerazioni svolte ad un esempio.

La funzione $\cot x$ ha per poli semplici, coi residui eguali all'unità, $\mathrm{i}$ punti

$$
\pm l \pi . \quad(l=0,1,2,3, \ldots)
$$

Nell'intorno dell'origine ha lo sviluppo:

$$
\cot x=\frac{\mathrm{I}}{x}-\frac{4^{B_{2} x^{2}}}{\mathrm{I} .2}+\frac{4^{2} B_{4} x^{4}}{\mathrm{I} \cdot 2 \cdot 3 \cdot 4}-\ldots
$$

in cui $B_{2}, B_{4}, \ldots$ sono $\mathrm{i}$ numeri di Bernoulli (*).

La sommı dei residui della funzione

$$
\frac{\cot x}{x}
$$

non è convergente, dunque $\left(\mathrm{n}^{\circ} 4\right)$ il minimo valore di $m$ non puó essere inferiore ad $\mathrm{I}$; ma $m$ puó scegliersi eguale ad $\mathrm{I}$, perchè si puó dimostrare che, posto

$$
R_{k}=k \pi+\frac{\pi}{2},
$$

|cot $x \mid$ resta sul contorno $C_{k}$ minore d'un numero fisso $M$, comunque grande sia $k\left({ }^{* *}\right)$.

La funzione $\cot x$ ha dunque lo sviluppo principale di $I^{\circ}$ ordine, che, in questo caso coincide con quello di Hermite:

$$
\cot x=\frac{\mathrm{I}}{x}+\sum\left(\frac{\mathrm{I}}{x-l \pi}+\frac{\mathrm{I}}{l \pi}\right) .
$$

Per questa via si ha il vantaggio di aver determinato la funzione intera, che resta incognita con quel metodo, e che si riduce alla costante zero.

(*) Cfr. Ce sà r o, Analisi algebrica, pag. 282.

(*) Picard, Traité d'Analyse, t. II, p. 165. 
SULlLO SVILUPPO D'UNA FUNŻioNe UNIFORME, ETC.

Iót

La considerazione che la serie dei residui della funzione

$$
\frac{\cot x}{x^{2 \mu}}
$$

è nulla, porta alle note relazioni $(*)$ :

$$
s_{2 \mu}=(-1)^{\mu-1} \frac{2^{2 \mu-x} B_{2 \mu} \pi^{2 \mu}}{(2 \mu) !}
$$

ove

$$
s_{2 \mu}=\sum_{k=1}^{\infty} \frac{\mathrm{I}}{k^{2 \mu}}
$$

8. Faremo ancora qualche osservazione.

Quando una funzione $f(x)$ ammette uno sviluppo d'ordine finito $m$, caratterizzato da certi polinomi $P_{l}(x)$ di grado $m-1$ in $x$, nel qual caso essa ammetterà anche lo sviluppo (6), è evidente che nel fare la derivata $m^{\text {ima }}$ di $f(x)$, i polinomi $P_{l}$ spariscono; sicchè la serie formata colle derivate $m^{\text {ime }}$ delle caratteristiche di $f(x)$ è convergente uniformemente in tutto il piano, e si ha

$$
f^{(m)}(x)=\sum_{i=0}^{\infty} G_{l}^{(m)}\left(\frac{\mathbf{I}}{x-a_{l}}\right)
$$

Di qui concludiamo subito, immaginando trasportata al primo membro la quantità $G_{0}^{(m)}\left(\frac{\mathrm{I}}{x}\right)$ corrispondente al valore zero dell'indice, e ponendo $x=0$, che è convergente la serie

$$
\sum_{i=3}^{\infty} G_{l}^{(m)}\left(\frac{1}{-a_{l}}\right)
$$

Analoghe conclusioni si hanno facendo le derivate di ordine

(*) Cesazro, 1. c. pag. 48 t. 
superiore ad m; dunaue le serie

$$
\sum_{m=1}^{\infty} G_{l}^{(v)}\left(\frac{1}{-a_{l}}\right) \quad(v \geq m)
$$

sono convergenti.

Or si puo vedere facilmente che la serie (7), per ogni dato $\vee \supseteq m$, è la somma dei residui relativi ai punti $a_{b}$, esclusa l'origine $a_{o}$, della funzione

$$
\frac{f(z)}{z^{v+z}}
$$

a meno d'un fattor numerico finito.

Avevamo appunto osservato aglla fine del $n^{\circ} 3$ che tali serie per $\nu \supseteq m$ sono convergenti.

Suppongasi che le singolarità della funzione $f(x)$ siano singolaritạ polari di prim'ordine, . sicehè si abbia:

$$
G_{l}\left(\frac{\mathrm{I}}{x-a_{l}}\right)=\frac{R_{l}}{x-a_{l}}
$$

In tal caso le serie (7) sono, a mepa d'un fattore numerica,

$$
\sum_{m=1}^{\infty} \frac{R_{l}}{a_{l}^{(+1}}: \quad(v \geq m)
$$

Tali serie adunque debbono esser convergenti, se la funzione deve avere uno sviluppo d'ordine finita $m_{+}$. Se poi si fa l'ipotesi che sia convergente la serie

$$
\sum_{m=1}^{\infty}\left|\frac{R_{l}}{a_{l}^{v+1}}\right| \text { per } v=m,
$$

allora, come ha dimostrato Hermite, la funzione ammette uno sviluppo d'ordine finito $m$; e precisamente, posto

$$
P_{l}(x)=\sum_{\nu=0}^{m-x} \frac{x^{\nu}}{a_{l}^{\nu+x}}
$$


si ha :

$$
f(x)=g(x)+\sum_{l=0}^{\infty} R_{l}\left[\frac{1}{x-a_{l}}+P_{l}(x)\right] .
$$

Or bene questo sviluppo coincide collo sviluppo principale (6), poichè si trova facilmente

$$
R_{l} P_{l}(x)=\sum_{v=0}^{m-1} \sigma_{\nu+1, l} x^{\nu}
$$

Dunque, quando ad una funzione, dotata di singolarità polari

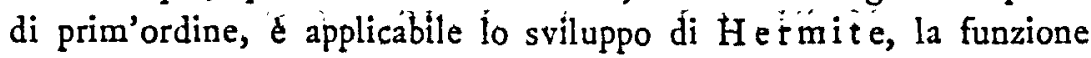
intera che completa lo sviluppo è data $\left[\mathrm{n}^{\circ} 5,(6)\right]$ da

$$
g(x)=\sum_{y=-m}^{\infty} x^{\nu} \sum_{k=0}^{\infty} \sigma_{y+x_{1}, *}
$$

Palermo, 28 febbrajo 1897 .

F. BuCCA. 